\title{
Perda de peso em pacientes oncológicos: prevalência e prognóstico relacionados a sexo, idade, localização do tumor e sintomas de impacto nutricional
}

\author{
Weight loss in cancer patients: prevalence and prognosis related to sex, age, tumor site and \\ nutritional impact symptoms
}

DOI: $10.37111 /$ braspenj.2020351014

Dalton Luiz Schiessel'

Amanda Kamitani Góis Orrutéa ${ }^{2}$

Sabrina Eduarda da Silva²

Mariana Abe Vicente Cavagnari ${ }^{3}$

Caryna Eurich Mazur ${ }^{4}$

Diogo Dequech Gavarrete ${ }^{5}$

Lindsay Bianca Buzato Antunes ${ }^{6}$

\section{Unitermos:}

Neoplasias. Desnutrição. Avaliação nutricional. Intomas de impacto nutricional.

\section{Keywords:}

Cancer. Malnutrition. Nutritional assessment. Nutritional impact symptoms.

\section{Endereço para correspondência:}

Dalton Luiz Schiessel

Universidade Estadual do Centro-Oeste

Campus CEDETEG - Departamento de Nutrição.

Alameda Élio Antonio Dalla Vecchia, 838 - Vila Carli

- Guarapuava, PR, Brasil - CEP 85040-167

E-mail: daltonls68@gmail.com

\section{Submissão}

15 de setembro de 2019

Aceito para publicação

27 de março de 2020

\begin{abstract}
RESUMO
Introdução: O câncer causa aumento nas demandas nutricionais e a presença de sintomas de impacto nutricional (SIN) contribuem para redução da ingestão e absorção de nutrientes, levando à perda de peso, desnutrição e predizer a sobrevida global. Avaliar a prevalência e predizer a perda de peso relacionada ao câncer, ao Esquema de Grade e aos SIN. Método: Foram coletados dados de 2012 a 2018 da primeira consulta nutricional de pacientes oncológicos em clínica vinculada ao SUS na cidade de Guarapuava-PR. O desfecho primário foi determinar o \% de perda de peso (\%PP), SNI e pelo Esquema de Grade proposta por Martin et. al (2015) foi determinado o prognóstico pela análise de Regressão Logística Multinomial (RLM) univariada e multivariada (ajustado para idade, sexo e localização do tumor). Resultados: 1164 pacientes com idade de 56,9 anos. Na primeira consulta foi observado um 6,7 de \%PP e foi observado que $21,6 \%$ dos pacientes eram baixo peso. As principais localizações e \%PP foram respectivamente: Pulmãol 40 $(12,0 \%)$ e $\%$ PP $9,4 \%$, Cabeça e Pescoço $113(9,7 \%)$ e $\%$ PP 10,5\%, Colorretal 84(7,2\%) e \%PP 10,3\%, Estômago 90(7,7\%) e \%PP 13,7\%, Esôfago 85(7,3\%) e \%PP 14,0\%, Pâncreas 24(2,1\%) e $\% \mathrm{PP} 16,1 \%$. Os principais SIN foram: xerostomia $(51,0 \%)$, dor abdominal $(23,0 \%)$, constipação $(21,7 \%)$, náusea $(15,3 \%)$ e alteração do paladar $(10,5 \%)$. Na RML para a análise univariada a idade, sexo, localizações de câncer e os SIN e para a análise multivariada todas a localizações do câncer apresentaram significativo OR para as serem classificados na grade 3 e 4 . Conclusão: Antes da quimioterapia a perda de peso e desnutrição estão presentes. A localização do câncer e SIN aumentam a chance do paciente ser classificado na grade 3 e 4 , levando estes pacientes a um pior estado nutricional e contribuindo para os resultados adversos.
\end{abstract}

\section{ABSTRACT}

Introduction: Cancer causes an increase in nutritional demands and the presence of of nutritional impact symptoms (NIS) contributes to reduction of nutrient intake and absorption, leading to weight loss, malnutrition and predicting overall survival. Assess the prevalence and predict the weight loss related to cancer, the Grade Scheme and the NIS. Methods: Data were collected from 2012 to 2018 from the first nutritional consultation of cancer patients in a clinic linked to SUS in the city of Guarapuava-PR. The primary outcome was to determine the $\%$ of weight loss (\% WL), NIS and by the Grade Scheme proposed by Martin et. al (2015) the prognosis was determined by univariate and multivariate Multinomial Logistic Regression (MLR) analysis (adjusted for age, sex and tumor location). Results: 1164 patients aged 56.9 years. In the first consultation, a $6.7 \% \mathrm{WL}$ was observed, and it was observed that $21.6 \%$ of the patients were underweight. The main sites and \%WL were, respectively: Lung $140(12.0 \%)$ and $9.4 \% \mathrm{WL}$, Head and Neck $113(9.7 \%)$ and $10.5 \% W L$, Colorectal 84 (7.2\%) and 10.3\%WL, Stomach $90(7.7 \%)$ and $13.7 \% W L$, Esophagus 85 $(7.3 \%)$ and $14.0 \% \mathrm{WL}$, Pancreas $24(2.1 \%)$ and $16.1 \% \mathrm{WL}$. The main NIS were: dry mouth $(51.0 \%)$, abdominal pain $(23.0 \%)$, constipation $(21.7 \%)$, nausea $(15.3 \%)$ and altered taste $(10.5 \%)$. In the RML for univariate analysis, age, sex, cancer site and SIN and for multivariate analysis, all cancer locations showed significant OR to be classified in grades 3 and 4 . Conclusion: Before chemotherapy, weight loss and malnutrition are present. The cancer site and SIN increase the chance of the patient being classified in grades 3 and 4 , leading these patients to a worse nutritional status and contributing to adverse results.

1. Nutricionista; Doutor em Biologia Celular e Molecular pela Universidade Federal do Paraná; Pós-Doutorado pela University of Alberta - Cross Cancer Institute, Edmonton - Canadá; Professor do Departamento de Nutrição da Universidade Estadual do Centro Oeste (UNICENTRO), Guarapuava, PR, Brasil.

2. Graduanda em Nutrição pela Universidade Estadual do Centro Oeste (UNICENTRO), Guarapuava, PR, Brasil.

3. Nutricionista; Doutora em Ciências pelo Programa de Pós-Graduação em Gastroenterologia pela Universidade Federal de São Paulo; Professora do Departamento de Nutrição da Universidade Estadual do Centro Oeste (UNICENTRO), Guarapuava, PR, Brasil.

4. Nutricionista; Doutora em Medicina Interna e Ciências da Saúde pela Universidade Federal do Paraná; Professora do Departamento de Nutrição da Universidade Estadual do Centro Oeste (UNICENTRO), Guarapuava, PR, Brasil.

5. Médico Oncologista, Hospital de Caridade São Vicente de Paulo, Clínica de Oncologia, Guarapuava, PR, Brasil.

6. Nutricionista, Hospital de Caridade São Vicente de Paulo, Clínica de Oncologia, Guarapuava, PR, Brasil. 


\section{INTRODUÇ̃̃O}

A estimativa mundial mostra que, em 2012, ocorreram 14,1 milhões de casos novos de câncer e 8,2 milhões de óbitos pela doença. Para o Brasil, nos anos de 2018 e 2019, a estimativa foi de ocorrência por ano de 600 mil novos casos. As doenças e agravos não transmissíveis (DANT) são um importante problema de saúde pública em todo o mundo, sendo as neoplasias uma das principais causas de óbito da população mundial. A taxa de mortalidade entre populações pode variar, sendo a primeira principal causa de óbito no Canadá e a segunda nos Estados Unidos e Brasil ${ }^{1-3}$.

A "caquexia-associada ao câncer" (CAC) é uma síndrome caracterizada pela perda involuntária do peso corporal, que é conduzida por uma combinação variável de mudanças metabólicas (gasto energético elevado, excesso de catabolismo e inflamação) e redução da ingestão de alimentos, podendo levar os pacientes à desnutrição ${ }^{4}$. A CAC é um marcador de prognóstico desfavorável que afeta a maior parte dos pacientes. Durante a trajetória da doença leva ao aumento na perda de peso e representa causa direta de pelo menos $20 \%$ das mortes associadas ao câncer ${ }^{5}$.

Pacientes com CAC manifestam complicações relacionadas ao tratamento, apresentando alto risco de toxicidade e tolerando menos ciclos de quimioterapia, além de queda na qualidade de vida e redução da sobrevida ${ }^{6-9}$. A CAC não pode ser totalmente revertida pelo suporte nutricional convencional e leva ao comprometimento funcional progressivo, sendo um processo contínuo que é compreendido em três estágios de relevância clínica: pré-cachexia, caquexia e caquexia refratária ${ }^{6}$. Ela pode ser diagnosticada quando o paciente apresenta: a) perda de peso > 5\% nos últimos 6 meses (na ausência de fome simples); ou b) índice de massa corporal (IMC) $<20$ e qualquer grau de perda de peso $>2 \%{ }^{4}$ Além disso, a perda de peso aumenta os custos financeiros para os sistemas de saúde pública no manejo de pacientes, por conta do alto custo das hospitalizações prolongadas ${ }^{10}$. Porém, os sistemas de classificação de perda de peso não levam em conta o benéfico potencial de um maior peso corporal inicial na avaliação de risco prévio de pacientes ${ }^{8}$. Infelizmente, os clínicos muitas vezes subestimam o risco que a perda de peso representa, podendo afetar a trajetória de vida dos pacientes com câncer. $O$ que também não recebe atenção adequada por parte dos pacientes e seus cuidadores, mesmo quando o risco de desnutrição é reconhecido ${ }^{10}$, por considerarem como um efeito colateral normal relacionado à doença ${ }^{11}$.

Recentemente, Martin et al. ${ }^{8}$ propuseram uma classificação robusta que relaciona o percentual de perda de peso com IMC e faz um prognóstico de sobrevida risco na matriz (sobrevivência mais longa), e os graus 1, 2, 3 e 4 foram atribuídos aos subgrupos, de acordo com a diminuição da sobrevivência e aumento do risco de óbito. Os pacientes que no momento do diagnóstico foram classificados com grade 0 tiveram média de sobrevida de 20,9 meses; grade 1 corresponde à sobrevida média de 14,6 meses; grade 2 de 10,8 meses; grade 3 de 7,6 meses; e grade 4 com média de 4,3 meses de sobrevida. Essa classificação em grades foi recentemente recomendada pela European Society for Clinical Nutrition and Metabolism (ESPEN), para avaliar perda de peso em pacientes com câncer ${ }^{12}$.

De um modo geral, o câncer por si e seus tratamentos (quimio/radioterapia e/ou cirurgia) levam a alterações fisiológicas, comprometimentos funcionais progressivos e desenvolvimento de sintomas de impacto nutricional (SIN) que limitam ou impedem a alimentação e provocam efeitos negativos durante a trajetória da doença ${ }^{13}$. Anorexia e diminuição progressiva da ingestão de alimentos ${ }^{14}$ podem ocorrer por conta de alterações na deglutição (disfagia, odinofagia, xerostomia, alteração de paladar/olfato e mucosite), pela má absorção de nutrientes (vômitos e diarreia) e por alterações intestinais (saciedade precoce, dor, obstrução e constipação) ${ }^{10,15,16}$, que vão contribuir para a perda de peso e efeitos adversos ${ }^{17}$.

Uma das ferramentas utilizadas para avaliar os SIN é a Avaliação Global Subjetiva Gerada pelo Paciente (ASGPPP), que avalia sintomas que impedem ou dificultam o paciente de se alimentar, como anorexia, náuseas, vômitos, constipação, diarreia, feridas na boca, xerostomia (boca seca), alterações de paladar e/ou olfato, disfagia (dificuldade para engolir), odinofagia (dor ao deglutir), saciedade precoce e outros sintomas ${ }^{18}$. Em uma população com diferentes localizações de tumor, um estudo observou elevado número de pacientes com SIN que os impediam de comer. Os principais SIN apresentados foram anorexia (47\%), saciedade precoce (43\%), náuseas (20\%) e sabor anormal $(20 \%)^{13}$, contudo, na maioria dos pacientes, os sintomas eram relacionados à localização do tumor.

Câncer de cabeça e pescoço, esôfago e pulmão apresentam um espectro alto de SIN, podendo ocorrer disfagia, mucosite, dificuldades de mastigação e odinofagia $^{17}$, fazendo com que mais da metade dos pacientes tenha consumo alimentar reduzido e apresente grande 
perda de peso ${ }^{19}$. A presença de SIN pode levar à redução da ingestão calórica, evidenciando o quadro da CAC 20 .

Os cânceres do trato gastrointestinal (estômago, intestino e órgãos anexos) geralmente provocam SIN, como odinofagia, regurgitação, perda de peso e saciedade precoce. Perda de apetite, desconforto e dor abdominal, má digestão, tempo de trânsito intestinal reduzido e supercrescimento bacteriano também são frequentes nestes pacientes. No câncer de pâncreas, há também a insuficiência pancreática exócrina (incapacidade de secretar enzimas digestivas em quantidade suficiente). Em canceres gastrointestinais, é comum a presença de náuseas, vômitos, mal-estar e anorexia, fatores que intercorrem com a $\mathrm{CAC}^{21}$. $O$ câncer colorretal apresenta alguns sintomas em particular, por mudança no funcionamento intestinal, como constipação ou diarreia, anemia, cólica abdominal, sangramento pelo reto e sensação de evacuação incompleta, contribuindo para o desconforto intestinal e também para redução da ingestão alimentar, promovendo a perda de peso e contribuindo no processo da $\mathrm{CAC}^{13}$.

Desta forma, avaliar a incidência dos principais SIN relacionados à localização do tumor auxilia no manejo nutricional, possibilitando a utilização de estratégias que atuem melhorando o apetite e a capacidade de ingerir e digerir alimentos ${ }^{13}$. $O$ estado nutricional de pacientes com câncer merece maior atenção, para que possam ser evitadas complicações, como o desenvolvimento de $\mathrm{CAC}^{6}$. A utilização de estratégias nutricionais para atenuar a perda de peso e melhorar o estado nutricional pode contribuir para melhora na qualidade e quantidade de alimentos consumidos e, consequentemente, no tempo de sobrevida ${ }^{22}$. O presente estudo objetivou prognosticar a perda de peso prévia ao tratamento quimioterápico e correlacionar à presença de SIN em pacientes diagnosticados com câncer na região de Guarapuava.

\section{MÉTODO}

\section{Desenho do Estudo}

Estudo classificado como transversal de caráter retrospectivo, com dados coletados dos prontuários de 2012 a 2018 de primeira consulta nutricional de pacientes oncológicos, previamente ao tratamento quimioterápico, no Hospital São Vicente, na cidade de Guarapuava-PR. O estudo foi aprovado pelo Comitê de Ética em Pesquisa - COMEP/UNICENTRO/G, sob o protocolo de pesquisa de número 918.226 de 09/04/2014.

\section{Avaliação Antropométrica}

Foram coletados dados dos prontuários: demográficos (sexo e localização do tumor); antropométricos (peso usual, peso atual e estatura); e presença de SIN (obtidos através da ASG-PPP, aplicada pela nutricionista do hospital). Foi calculada a perda de peso, o percentual de perda de peso e o IMC (Organização Mundial da Saúde, 1998) e avaliada a perda de peso de acordo com os critérios do Esquema de Classificação de Grades, segundo Martin et al. ${ }^{8}$.

\section{Análise Estatística}

Os dados foram analisados por meio de estatística descritiva, com a utilização de médias, desvio padrão e frequências relativas e absolutas. Valores descritivos foram expressos em média $\pm S D$. Todos os valores de $p$ foram de dois lados, e níveis de significância são valores de $\leq$ 0,05 . Foi realizado teste de normalidade (Shapiro-Wilk) para verificar a distribuição das variáveis numéricas. A comparação das variáveis numéricas foi realizada por meio de teste $\dagger$ de Student ou teste de Mann-Whitney. A comparação das variáveis qualitativas foi realizada por meio do teste Qui-Quadrado Pearson, para determinar desfecho primário em determinar o \% de perda de peso de acordo com cada localização do câncer. $\bigcirc$ prognóstico foi realizado pela análise de regressão logística multinomial (RLM) os desfechos tendo a categoria de referência o grade 1 para determinar o odds ratio (OR) univariada e multivariada ajustado para idade, sexo, patologia e para SNI no programa SPSS ${ }^{\circledR} 22$.

\section{RESULTADOS}

A amostra total foi de 1164 pacientes, sendo composta de $44,2 \%(n=515)$ do sexo masculino e $55,8 \%(n=649)$ do sexo feminino (Tabela 1). A população foi constituída por $46,7 \%$ de idosos (<60 anos), apresentando média de idade de 56,9 $\pm 13,0$ anos. Notou-se que os pacientes apresentaram redução significativa do peso usual (PU) relatado pelos indivíduos para o peso inicial (PI), aferido na consulta nutricional precedente ao tratamento de quimioterapia. Consequentemente, houve perda de peso média de $6,1 \pm 12,0 \%$ do peso corporal, causando redução do IMC, assim houve um aumento de 2,4; 2,9 e 2,0 vezes na incidência de baixo peso para todos os pacientes e para o sexo masculino e feminino, respectivamente, revelando alteração significativa da classificação do estado nutricional. Comparando entre os sexos, observou-se que os 
Tabela 1 - Caracterização da amostra e alterações de peso de pacientes oncológicos, Guarapuava (PR), 2012-2018.

\begin{tabular}{|c|c|c|c|c|c|}
\hline & Geral & $\mathbf{P}$ & Masculino & Feminino & $p$ \\
\hline n (\%) & $1164(100)$ & & $515(44,2)$ & $649(55,8)$ & \\
\hline \multicolumn{6}{|l|}{ Peso (kg) } \\
\hline Inicial & $65,5 \pm 15,8$ & & $67,5 \pm 15,5$ & $63,9 \pm 15,8$ & $<0,001^{* *}$ \\
\hline Altura (m) & $1,65 \pm 0,1$ & & $1,71 \pm 0,1$ & $1,59 \pm 0,1$ & $<0,001^{\star *}$ \\
\hline \multicolumn{6}{|l|}{ IMC (kg/m²) } \\
\hline Inicial & $24,1 \pm 5,5$ & & $22,9 \pm 4,5$ & $25,1 \pm 5,9$ & $<0,001^{* *}$ \\
\hline \multicolumn{6}{|c|}{ Mudança de Peso } \\
\hline$\%$ & $-6,1 \pm 12,0$ & & $-9,2 \pm 11,0$ & $-4,8 \pm 12,3$ & $<0,001^{\star *}$ \\
\hline \multicolumn{6}{|c|}{ IMC Usual - n (\%) } \\
\hline Baixo peso & $99(9,0)$ & $<0,001 \#$ & $44(8,9)$ & $55(9,0)$ & \\
\hline \multicolumn{6}{|c|}{ IMC Inicial - n (\%) } \\
\hline Baixo peso & $238(21,7)$ & & $132(26,0)$ & $115(18,0)$ & \\
\hline Eutrofia & $454(41,3)$ & & $234(46,2)$ & $243(38,0)$ & $<0,001 \# \#$ \\
\hline Sobrepeso & $253(23,0)$ & & $100(19,7)$ & $160(25,0)$ & \\
\hline Obesidade & $154(14,0)$ & & $41(8,1)$ & $121(18,9)$ & \\
\hline
\end{tabular}

Tabela 2 - Localização do câncer e percentual de mudança de peso, Guarapuava (PR), 2012-2018.

\begin{tabular}{lcc}
\hline Localização & $\mathbf{n}(\%)$ & $\% \mathbf{M P}$ \\
\hline Pâncreas & $24(2,1 \%)$ & $-16,2 \pm 11,3$ \\
Esôfago & $85(7,3 \%)$ & $-14,0 \pm 12,3$ \\
Estômago & $90(7,7 \%)$ & $-13,7 \pm 12,7$ \\
Colorretal & $84(7,2 \%)$ & $-10,3 \pm 8,2$ \\
Cabeça e Pescoço & $113(9,7 \%)$ & $-10,5 \pm 11,2$ \\
Pulmão & $140(12,0 \%)$ & $-9,3 \pm 10,0$ \\
Outros & $111(9,5 \%)$ & $-6,9 \pm 10,8$ \\
Linfoma & $55(4,7 \%)$ & $-6,3 \pm 10,1$ \\
Aparelho Reprodutor Feminino & $112(9,6 \%)$ & $-5,3 \pm 11,7$ \\
Aparelho Reprodutor Masculino & $60(5,2 \%)$ & $-2,0 \pm 9,8$ \\
Mama & $290(24,9 \%)$ & $0,4 \pm 10,6$ \\
\hline
\end{tabular}

$\mathrm{n}=$ número amostral; \%MP= percentual de mudança de peso.

achados são significativamente diferentes para todos os parâmetros, com especial atenção para o \% de perda de peso corporal que foi maior para homens em comparação às mulheres $(9,2 \%$ vs. $4.8 \%$, respectivamente). A Tabela 2 apresenta a localização do câncer e \% de mudança de peso.

Utilizando os dados de IMC-I e \%MP, foi realizada a classificação dos pacientes de acordo com o esquema de grades de Martin et al. ${ }^{8}$. Com esses dados foi feita a caracterização dos pacientes de cada grade (Tabela 3). Grande parte dos pacientes encontra-se em G0 com 37,1\%, subgrupo de menor risco e sobrevida mais longa. Porém, em segundo foi $\mathrm{G} 4$ com $31 \%$, subgrupo de maior risco e sobrevida mais curta. A média de idade de G0 $(53,8$ $\pm 13,3$ anos) foi estatisticamente menor que de G4 (59,3 $\pm 12,7$ anos).

O PU de G4 $(67,6 \pm 13,4 \mathrm{~kg})$ foi o menor de todos os grades, seguido de $\mathrm{G} 0(69,2 \pm 15,1 \mathrm{~kg})$, não havendo diferença estatística entre eles. O IMC-U de G4 (24,7 \pm $4,4 \mathrm{~kg} / \mathrm{m}^{2}$ ) foi significativamente menor que de todas os outros grades e o de $G 0\left(25,8 \pm 5,3 \mathrm{~kg} / \mathrm{m}^{2}\right)$ apresentou o segundo menor valor. O PI de G4 $(54,2 \pm 10,1 \mathrm{~kg})$ foi estatisticamente menor que de todos os outros grades. $O$ IMC-I foi significativamente menor em G4 (19,8 \pm 7,8 kg/ $\left.\mathrm{m}^{2}\right)$ do que nos outros grades. A perda de peso foi significativamente maior em G4 (-19,3 \pm 7,8\%), decrescendo a cada grade, sendo que em G0 a média foi positiva, representando ganho de peso.

Com relação à localização do câncer, o mais frequente foi o de mama $(25,1 \%)$, seguido de pulmão $(11,8 \%)$ e cabeça e pescoço $(9,8 \%)$. O maior percentual de perda de peso foi observado naqueles localizados no pâncreas $(-16,15 \pm 11,29 \%)$, seguido de esôfago $(-14,00 \pm 12,33 \%)$ e estômago $(-13,73 \pm 12,68 \%)$. Houve média de ganho de peso nos casos de câncer de mama $(0,43 \pm 10,59 \%)$. 
Tabela 3 - Caracterização dos pacientes classificados no esquema de grades de Martin et al. ${ }^{8}$, Guarapuava (PR), 2012-2018.

\begin{tabular}{|c|c|c|c|c|c|}
\hline & Grade 0 & Grade 1 & Grade 2 & Grade 3 & Grade 4 \\
\hline $\mathrm{n}(\%)$ & $409(37,1)$ & $54(4,9)$ & $108(9,8)$ & $190(17,2)$ & $342(31,0)$ \\
\hline Idade (anos) & $53,8 \pm 13,3$ & $57,9 \pm 11,0$ & $56,4 \pm 11,8$ & $57,9 \pm 12,9^{*}$ & $59,3 \pm 12,7 \#$ \\
\hline \multicolumn{6}{|l|}{ Peso (kg) } \\
\hline Usual & $69,2 \pm 15,1$ & $79,6 \pm 19,0$ & $72,9 \pm 13,8$ & $73,8 \pm 15,6$ & $67,6 \pm 13,4$ \\
\hline Inicial & $72,0 \pm 15,6$ & $77,0 \pm 18,1$ & $69,0 \pm 12,4$ & $66,2 \pm 12,6$ & $54,2 \pm 10,1$ \\
\hline Altura & $1,64 \pm 0,1$ & $1,65 \pm 0,1$ & $1,64 \pm 0,1$ & $1,66 \pm 0,1$ & $1,65 \pm 0,1$ \\
\hline \multicolumn{6}{|l|}{$\operatorname{IMC}\left(\mathrm{kg} / \mathrm{m}^{2}\right)$} \\
\hline Usual & $25,8 \pm 5,3$ & $29,2 \pm 6,0$ & $27,1 \pm 4,6$ & $26,6 \pm 4,9$ & $24,7 \pm 4,4$ \\
\hline Inicial & $26,9 \pm 5,4$ & $28,2 \pm 5,5$ & $25,6 \pm 4,0$ & $23,9 \pm 3,8$ & $19,8 \pm 3,1$ \\
\hline \multicolumn{6}{|c|}{ Mudança de Peso } \\
\hline$\%$ & $4,5 \pm 7,9$ & $-3,1 \pm 1,5$ & $-5,2 \pm 1,9$ & $-9,8 \pm 3,4$ & $-19,3 \pm 7,8$ \\
\hline \multicolumn{6}{|c|}{ IMC Usual - n (\%) } \\
\hline Baixo peso & $43(10,6)$ & - & - & $15(7,9)$ & $41(12,0)$ \\
\hline Eutrofia & $160(39,5)$ & $19(35,2)$ & $42(38,9)$ & $53(27,9)$ & $162(47,4)$ \\
\hline Sobrepeso & $115(28,4)$ & $7(13,0)$ & $42(38,9)$ & $85(44,7)$ & $94(27,5)$ \\
\hline Obesidade & $87(21,5)$ & $28(51,9)$ & $24(22,2)$ & $37(19,5)$ & $45(13,2)$ \\
\hline \multicolumn{6}{|c|}{ IMC Inicial - n (\%) } \\
\hline Baixo peso & $30(7,4)$ & - & - & $21(11,1)$ & $187(54,7)$ \\
\hline Eutrofia & $135(33,3)$ & $22(40,7)$ & $55(50,9)$ & $108(56,8)$ & $134(39,2)$ \\
\hline Sobrepeso & $131(32,3)$ & $11(20,4)$ & $39(36,1)$ & $51(26,8)$ & $21(6,1)$ \\
\hline Obesidade & $109(26,9)$ & $21(38,9)$ & $14(13,0)$ & $10(5,3)$ & - \\
\hline
\end{tabular}

Teste ANOVA com pós teste de Tukey * G0 x G4 p=<0,003; G0 x G4 p=<0,001.

$\mathrm{n}=$ número amostral; $\mathrm{p}=$ significância do teste estatístico; \%= percentual; $\mathrm{PU}=$ peso usual; $\mathrm{Pl}=$ peso inicial; $I \mathrm{MC}-\mathrm{U}=$ índice de massa corpórea usual; IMC-I= índice de massa corpórea inicial.

Tabela 4 - Análise da regressão logística multinomial univariada correlacionada com a classificação pelo sistema de grades de Martin et al. ${ }^{8}$, Guarapuava (PR), 2012-2018.

\begin{tabular}{|c|c|c|c|c|c|c|c|c|c|}
\hline \multirow{2}{*}{$\begin{array}{l}\text { Parâmetros } \\
\text { correlatos }\end{array}$} & \multirow{2}{*}{$\begin{array}{c}\mathrm{N} \text { de pacientes } \\
\text { na análise } \\
\mathrm{n}(\%)\end{array}$} & \multicolumn{8}{|c|}{ Univariada } \\
\hline & & $\begin{array}{c}\text { Grade } 1 \\
\text { OR (95\% IC) }\end{array}$ & $P$ & $\begin{array}{c}\text { Grade } 2 \\
\text { OR }(95 \% \text { IC) }\end{array}$ & $P$ & $\begin{array}{c}\text { Grade } 3 \\
\text { OR (95\% IC) }\end{array}$ & $P$ & $\begin{array}{c}\text { Grade } 4 \\
\text { OR (95\% IC) }\end{array}$ & $P$ \\
\hline Idade & 1098 (100\%) & & & & & & & & \\
\hline \multicolumn{10}{|l|}{ Sexo } \\
\hline \multicolumn{10}{|l|}{ Patologias } \\
\hline Pâncreas & $24(2,2 \%)$ & 12,06 & 0,016 & 3,33 & 0,333 & 30,47 & $<0,001$ & 62,72 & $<0,001$ \\
\hline Esôfago & $77(7,0 \%)$ & $\begin{array}{c}(1,59-91,41) \\
4,52\end{array}$ & 0,037 & $\begin{array}{c}(0,29-37,87 \\
4,16\end{array}$ & 0,018 & $\begin{array}{c}(5,75-161,60) \\
13,97\end{array}$ & $<0,001$ & $\begin{array}{c}(13,20-297,98) \\
60,31\end{array}$ & $<0,001$ \\
\hline Estômago & $88(8,8 \%)$ & $\begin{array}{c}(1,10-18,74) \\
4,39\end{array}$ & 0,021 & $\begin{array}{c}(1,27-13,58) \\
3,02\end{array}$ & 0,054 & $\begin{array}{c}(5,01-38,94) \\
23,01\end{array}$ & $<0,001$ & $\begin{array}{c}(25,31-144,96) \\
37,72\end{array}$ & $<0,001$ \\
\hline Pulmão & $130(11,8 \%)$ & $\begin{array}{c}(0,60-6,23) \\
3,33\end{array}$ & 0,012 & $\begin{array}{c}(0,60-4,22) \\
3,67\end{array}$ & $<0,001$ & $\begin{array}{c}(4,26-18,77) \\
7,71\end{array}$ & $<0,001$ & $\begin{array}{c}(10,13-38,24) \\
18,30\end{array}$ & $<0,001$ \\
\hline Outros & $104(9,4 \%)$ & $\begin{array}{c}(1,31-8,47) \\
0,57 \\
(0,127-2,59\end{array}$ & 0,471 & $\begin{array}{c}(1,78-7,58) \\
1,59 \\
(0,72-3,50)\end{array}$ & 0,255 & $\begin{array}{c}(3,72-15,95) \\
4,60 \\
(2,24-9,42)\end{array}$ & $<0,001$ & $\begin{array}{c}(9,61-34,84) \\
7,12 \\
(3,70-13,70)\end{array}$ & $<0,001$ \\
\hline Linfoma & $50(9,4 \%)$ & - & - & 2,22 & 0,119 & 6,77 & $<0,001$ & 7,50 & $<0,001$ \\
\hline $\begin{array}{l}\text { Aparelho Reprodutor } \\
\text { Feminino }\end{array}$ & $107(9,7 \%)$ & $\begin{array}{c}1,40 \\
(0,49-4,04)\end{array}$ & 0,530 & $\begin{array}{c}(0,81-6,05) \\
2,02 \\
(0,97-4,19)\end{array}$ & 0,062 & $\begin{array}{c}(2,84-16,15) \\
4,25 \\
(2,06-8,77)\end{array}$ & $<0,001$ & $\begin{array}{c}(3,25-17,32) \\
6,28 \\
(3,24-12,18)\end{array}$ & $<0,001$ \\
\hline $\begin{array}{l}\text { Aparelho Reprodutor } \\
\text { Masculino } \\
\text { Mama }\end{array}$ & $\begin{array}{l}57(5,2 \%) \\
277(25,1)\end{array}$ & $\begin{array}{c}2,68 \\
(0,97-7,44) \\
-\end{array}$ & 0,058 & $\begin{array}{c}1,23 \\
(0,44-3,46)\end{array}$ & 0,691 & $\begin{array}{c}5,27 \\
(2,37-11,71)\end{array}$ & $<0,001$ & $\begin{array}{c}1,79 \\
(0,92-5,15)\end{array}$ & 0,283 \\
\hline
\end{tabular}


Tabela 5 - Análise da regressão logística multinomial multivariada correlacionada com a classificação pelo sistema de grades de Martin et al. ${ }^{8}$, Guarapuava (PR), 2012-2018.

\begin{tabular}{|c|c|c|c|c|c|c|c|c|c|}
\hline \multirow{2}{*}{$\begin{array}{l}\text { Parâmetros } \\
\text { correlatos }\end{array}$} & \multirow{2}{*}{$\begin{array}{c}\mathrm{N} \text { de pacientes } \\
\text { na análise } \\
\mathrm{n}(\%)\end{array}$} & \multicolumn{8}{|c|}{ Univariada } \\
\hline & & $\begin{array}{c}\text { Grade } 1 \\
\text { OR }(95 \% \text { IC) }\end{array}$ & $P$ & $\begin{array}{c}\text { Grade } 2 \\
\text { OR (95\% IC) }\end{array}$ & $P$ & $\begin{array}{c}\text { Grade } 3 \\
\text { OR }(95 \% \text { IC) }\end{array}$ & $P$ & $\begin{array}{c}\text { Grade } 4 \\
\text { OR }(95 \% \text { IC) }\end{array}$ & $P$ \\
\hline \multirow[t]{2}{*}{ Idade } & 1098 (100\%) & & & & & & & & \\
\hline & & $\begin{array}{c}1,019 \\
(0,99-1,04)\end{array}$ & 0,128 & $\begin{array}{c}1,010 \\
(0.99-1,03)\end{array}$ & 0,246 & $\begin{array}{c}1,016 \\
(1,002-1,031)\end{array}$ & 0,026 & $\begin{array}{c}1,020 \\
(1,007-1,034)\end{array}$ & 0,003 \\
\hline \multicolumn{10}{|l|}{ Sexo } \\
\hline Feminino & $612(55,5 \%)$ & & & - & & - & & 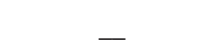 & \\
\hline Masculino & $491(44,5 \%)$ & $\begin{array}{c}1,32 \\
(0,52-3,32)\end{array}$ & 0,557 & $\begin{array}{c}1,10 \\
(0,58-2,08)\end{array}$ & 0,780 & $\begin{array}{c}1,044 \\
(0,62-1,74)\end{array}$ & 0,871 & $\begin{array}{c}0,95 \\
(0,61-1,49)\end{array}$ & 0,828 \\
\hline \multicolumn{10}{|l|}{ Patologias } \\
\hline Pâncreas & $24(2,2 \%)$ & \multirow{6}{*}{$\begin{array}{c}9,39 \\
(1,11-79,22) \\
3,28 \\
(0,67-16,13) \\
3,33 \\
(0,80-13,87) \\
3,49,14 \\
(0,86-144,08) \\
1,42 \\
(0,33-6,03) \\
2,51 \\
(0,86-7,35)\end{array}$} & 0,039 & \multirow{6}{*}{$\begin{array}{c}3,03 \\
(0,26-35,89) \\
3,66,13) \\
(1,02-13,11) \\
2,73 \\
(0,81-9,16) \\
6,66 \\
(2,47-17,97) \\
1,43 \\
(0,46-4,43) \\
3,25 \\
(1,46-7,25)\end{array}$} & 0,379 & \multirow{6}{*}{$\begin{array}{c}28,17 \\
(5,12-154,87) \\
12,26 \\
(4,10-36,60) \\
20,97 \\
(8,34-52,72) \\
18,89 \\
(7,52-47,46) \\
8,21 \\
(3,43-19,64) \\
6,39 \\
(2,93-14,00)\end{array}$} & $<0,001$ & \multirow{6}{*}{$\begin{array}{c}60,94 \\
(12,45-298,22) \\
55,20 \\
(21,62-140,96) \\
35,89 \\
(15,14-85,04) \\
27,32 \\
(11,43-65,28) \\
19,38 \\
(8,93-42,08) \\
16,18 \\
(8,16-32,08)\end{array}$} & $<0,001$ \\
\hline Esôfago & $77(7,0 \%)$ & & 0,143 & & 0,047 & & $<0,001$ & & $<0,001$ \\
\hline Estômago & $88(8,8 \%)$ & & 0,098 & & 0,105 & & $<0,001$ & & $<0,001$ \\
\hline Colorretal & $81(7,3 \%)$ & & 0,081 & & $<0,001$ & & $<0,001$ & & $<0,001$ \\
\hline Cabeça e Pescoço & $108(9,8 \%)$ & & 0,633 & & 0,535 & & $<0,001$ & & $<0,001$ \\
\hline Pulmão & $130(11,8 \%)$ & & 0,092 & & 0,004 & & $<0,001$ & & $<0,001$ \\
\hline Outros & $104(9,4 \%)$ & $\begin{array}{c}0,46 \\
(0,09-2,35)\end{array}$ & 0,349 & $\begin{array}{c}1,46 \\
(0,60-3,58)\end{array}$ & 0,404 & $\begin{array}{c}4,30 \\
(1,95-9,48)\end{array}$ & $<0,001$ & $\begin{array}{c}6,97 \\
(3,41-14,22)\end{array}$ & $<0,001$ \\
\hline Linfoma & $50(9,4 \%)$ & - & - & 2,16 & 0,156 & 6,83 & $<0,001$ & 7,95 & $<0,001$ \\
\hline Aparelho Reprodutor & $107(9,7 \%)$ & 1,41 & 0,522 & $\begin{array}{c}(0,74-6,29) \\
2,03\end{array}$ & 0,059 & $\begin{array}{c}(2,72-17,09) \\
4,30\end{array}$ & $<0,001$ & $\begin{array}{c}(3,30-17,32) \\
6,37\end{array}$ & $<0,001$ \\
\hline $\begin{array}{l}\text { Feminino } \\
\text { Aparelho Reprodutor } \\
\text { Masculino }\end{array}$ & $57(5,2)$ & $\begin{array}{c}(0,49-4,07) \\
1,90 \\
(0,49-7,96)\end{array}$ & 0,378 & $\begin{array}{c}(0,97-4,22) \\
1,25 \\
(0,37-4,24)\end{array}$ & 0,719 & $\begin{array}{c}(2,08-8,89) \\
5,26 \\
(1,99-13,89)\end{array}$ & $<0,001$ & $\begin{array}{c}(3,28-12,39) \\
2,11 \\
(0,66-6,74)\end{array}$ & 0,206 \\
\hline Mama & $277(25,1)$ & - & & - & & - & & - & \\
\hline
\end{tabular}

Interceptação em todas as análises $p<0,001$. A categoria de referência é: grade 0 .

$\mathrm{Na}$ Tabela 4, encontram-se os dados da análise estatística de RLM univariada, que correlaciona os grades com idade, sexo e localização do tumor. Com relação à idade, tendo como referência G0, a cada ano aumenta em 3,5\% a probabilidade de um paciente estar classificado em G4. Com relação ao sexo, tendo referência mulheres, a chance de um paciente estar em G4 e ser homem é 3,16 vezes maior. Na Tabela 5, encontram-se os dados da análise estatística de RLM multivariada, independente de sexo e idade, o risco relativo de um paciente com câncer de pâncreas estar em G4 é 60,94 vezes maior que de câncer de mama. Em segundo lugar, em maior probabilidade de estar em G4 vem o câncer de esôfago, com chance de 55,2 vezes, seguido do câncer de estômago com risco 35,89 vezes maior de estar em G4 que do grupo referência.

Os principais SIN apresentados na Tabela 6 foram xerostomia $(51 \%)$, dor abdominal $(23 \%)$, constipação $(21,7 \%)$, náusea (15,3\%) e alteração do paladar (10,5\%). Na Tabela 6 , se encontram os dados da análise estatística de RLM univariada correlacionando os SIN com a classificação pelo sistema de grades de G0 a G4, tendo como categoria de referência $G 0$. Os pacientes classificados em G3 apresentaram alto risco relativo para presença de esofagite, disfagia, vômito, anorexia e alteração de paladar, fatores limitantes do consumo alimentar. Os pacientes classificados em G4, que são aqueles que apresentaram grande perda de peso e redução do IMC previamente ao tratamento quimioterápico, apresentaram significativo risco relativo para presença de todos os SIN investigados neste estudo.

\section{DISCUSSÃO}

O presente estudo demonstra o que outros estudos vêm confirmando ao longo dos anos e, assim, reafirma que a perda de peso em pacientes oncológicos é alta já no momento da primeira consulta nutricional antecedente à quimioterapia. Homens perdem mais peso que mulheres na média geral, contudo, alguns tipos de câncer mais agressivos e com uma característica mais catabólica fazem esta diferença aparecer, uma vez que aproximadamente 1/4 da população foi diagnosticada com câncer de mama, que historicamente apresenta um perfil de ganho de peso. 
Tabela 6 - Análise da regressão logística multinomial correlacionando o SIN com a classificação pelo sistema de grades de Martin et al.8, Guarapuava (PR), 2012-2018.

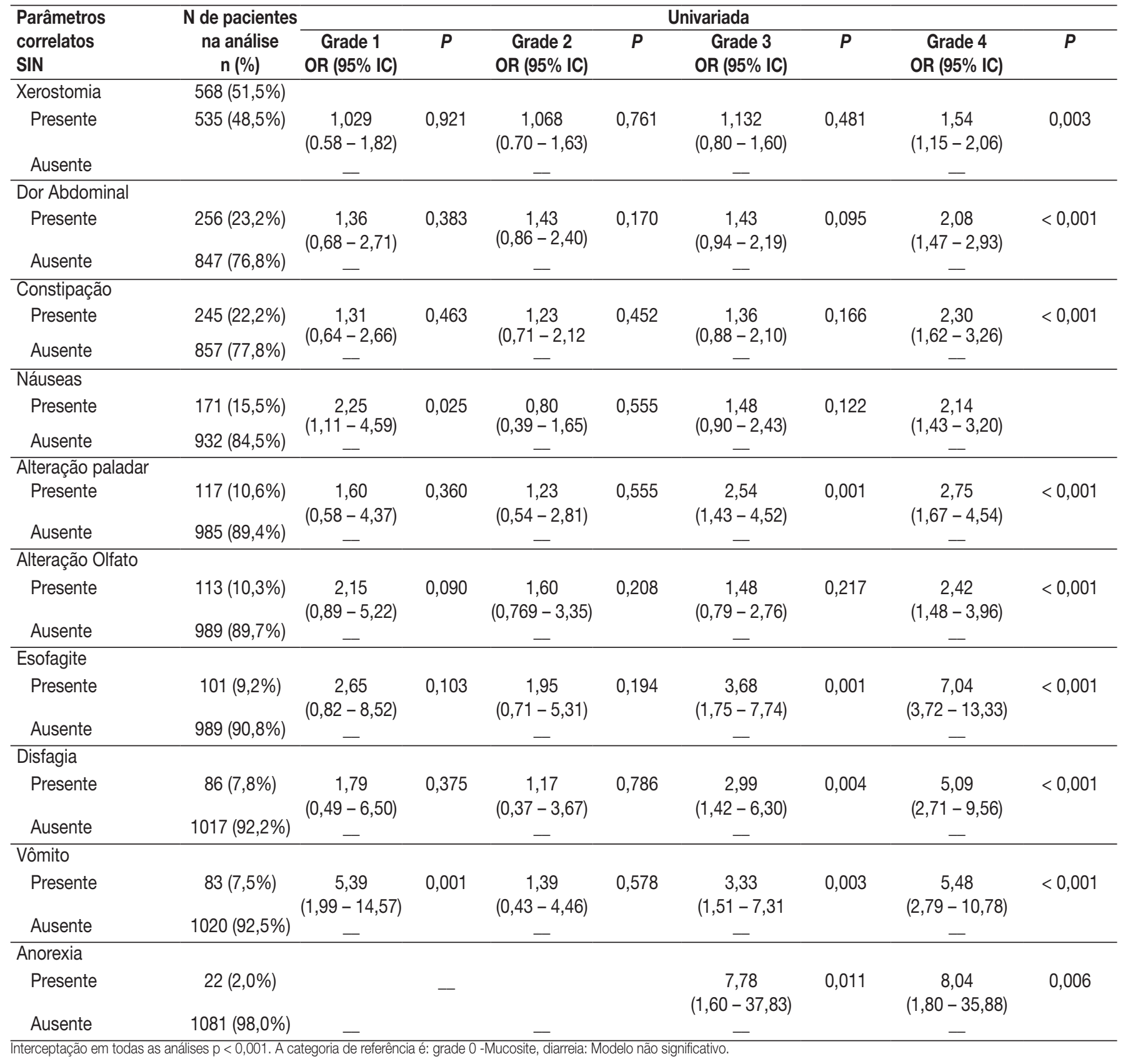

A abordagem de análise aplicada neste estudo, a RLM, apresenta robustez estatística consistente quando uma variável dependente é categórica e as variáveis explicativas são contínuas ou categóricas, fornecendo uma força analítica para determinar o risco relativo e predizer desfecho, sendo demonstrado que o parâmetro idade, patologias e SIN apresenta elevada chance dos pacientes serem qualificados nos grades 3 e 4 pela classificação do grade proposta por Martin et al. ${ }^{8}$.

A perda involuntária de peso é um dos sintomas da presença de doença, sendo que, na primeira consulta com
- oncologista, é observada prevalência de $25 \%$ a $70 \%$ de desnutrição relacionado à doença ${ }^{10}$ e, neste estudo, observou-se que o PU relatado pelo paciente foi significativamente maior que de Pl avaliado na primeira consulta com o profissional nutricionista, evidenciando que previamente ao tratamento quimioterápico há perda de peso de 6,1\% de peso corpóreo. Os homens apresentaram perda de peso $9,2 \%$ significativamente maior que as mulheres $4,8 \%$, o que pode ser explicado pela presença dos casos de câncer de mama, que tem como característica o predomínio de ganho de peso $^{23}$. 
A quantidade de indivíduos baixo peso pelo IMC-I foi 2,4 vezes maior que pelo IMC-U, ou seja, houve aumento significativo na quantidade de indivíduos nessa categoria se compararmos o peso que eles costumavam ter e o que passaram a apresentar antes de iniciarem o tratamento. Essa perda de peso pode ser explicada pela redução do consumo alimentar (relacionada à presença de SIN) e alterações metabólicas, como aumento do gasto energético em repouso, resistência à insulina, lipólise e depleção de proteína muscular, que podem ser geradas no organismo por conta do tumor ${ }^{12}$.

A perda de peso está relacionada diretamente com a capacidade do paciente em ingerir, digerir e metabolizar os alimentos. Desta forma, os tumores que afetam o trato gastrointestinal e respiratório são aqueles que apresentam maior perda de peso, como pode ser observado na Tabela 2. O subgrupo G4 foi o que apresentou maior perda de peso e G0 teve média de \% MP positiva, representando ganho de peso, o que pode ser explicado pelos casos de câncer de $m m^{24}$. As maiores perdas de peso foram observadas nos cânceres de pâncreas, esôfago e estômago, que fazem parte do TGI. Essa perda de peso pode ser diretamente relacionada às falhas na digestão e/ou absorção de nutrientes, que pode ser gerada pelo tumor que se localiza em uma das partes desse processo, além de ser comum nesses casos a presença de SIN, prejudicando o consumo alimentar e, consequentemente, o estado nutricional ${ }^{21}$.

Pelo sistema de grades de Martin et al. ${ }^{8}$, a maior parte dos pacientes se encontrava em G0 $(37,1 \%)$, seguido de G4 (31\%). Assim, boa parte dos pacientes se encontrava em um subgrupo de menor risco e maior tempo de sobrevida, porém uma parcela significativa estava no subgrupo de maior risco, merecendo maior atenção nutricional. $\bigcirc$ G4 é o subgrupo com maior perda de peso, consequentemente, apresentando maior risco de desenvolvimento de CAC, que pode levar a diversas complicações no tratamento e queda na qualidade de vida, além de diminuição no tempo de sobrevida ${ }^{4,8,9}$.

Pela análise estatística de RLM na univariada (Tabela 4), o parâmetro idade eleva a chance a cada ano em aumentar em 3,5\% de um paciente se encontrar na G4 e, na análise multivariada independente do sexo ou patologias, a chance para a idade elevar o RR foi $2 \%$ ao ano, ou seja, quanto mais velho o paciente, maior a chance de pior prognóstico e menor sobrevida. Desta forma, um cuidado maior deve ser direcionado aos pacientes idosos, contribuindo para prevenção do desenvolvimento de $\mathrm{CAC}^{16}$. Para as mesmas análises, o sexo não é significativo em interferir no RR para a classificação nos grades.

Analisando-se as patologias na RLM univariada, todas as patologias apresentaram elevado RR para os pacientes serem classificados nos Grades 3 e 4, assim como na análise multivariada. Os pacientes com o diagnóstico de canceres do trato gastrointestinal e pulmão apresentam elevado RR, uma vez que apresentam elevado \% de perda de peso, pois o critério para a classificação dos grades considera o IMC e a perda de peso para gradear os pacientes, contudo, isto indica reduzida sobrevida do paciente. Pacientes com câncer de mama geralmente apresentam ganho de peso e foram utilizados como referência para a análise do parâmetro. Os tumores do TGl, que estão diretamente relacionados com a utilização dos nutrientes pelo organismo, juntamente com a presença de SIN acabam levando à perda de peso em larga escala, por comprometimento do funcionamento do $\mathrm{TG}^{21}$.

Com relação aos SIN, o subgrupo G4 apresentou risco relativo para presença de todos os sintomas investigados no estudo, mesmo previamente ao tratamento quimioterápico. A presença de SIN dificulta a alimentação, podendo causar diminuição na ingestão calórica, levando a maior risco de desenvolvimento de CAC. Conhecer os sintomas apresentados pelo paciente é essencial para escolha de estratégias nutricionais que auxiliem na melhora do consumo alimentar e do estado nutricional ${ }^{20}$.

Sabendo que o estado nutricional pode afetar diretamente no tratamento, qualidade de vida e prognóstico de sobrevida de pacientes oncológicos, e observando a presença de perda de peso na maior parte dos cânceres, por alterações metabólicas ${ }^{6}$ e presença de SIN que influenciam no consumo alimentar ${ }^{13}$, fica evidente o papel fundamental do nutricionista no tratamento e acompanhamento destes indivíduos. Uma atenção ainda maior deve ser direcionada aos cânceres de TGI e aos idosos, pelo maior risco de perda de peso e desenvolvimento de CAC, podendo levar a prognósticos desfavoráveis ${ }^{5}$. A perda de peso ainda é subestimada pelos clínicos, pacientes e cuidadores $^{10}$. $\bigcirc$ acompanhamento nutricional de pacientes com câncer ainda é escasso e deveria receber maior atenção, de forma a contribuir para melhor resposta ao tratamento, prognóstico mais favorável e melhoria na qualidade de vida.

\section{CONCLUSÕES}

Previamente ao tratamento quimioterápico já é possível observar perda de peso em grande parte dos pacientes. Relacionando estado nutricional e perda de peso, com a classificação de grades de Martin et al., os piores prognósticos estavam presentes nos casos de câncer de pâncreas, esôfago e estômago, que fazem parte do TGI. A idade também foi observada como fator determinante para maiores perdas de peso. Da mesma forma, a presença de SIN pode prejudicar o estado nutricional, levando a maior risco de desenvolvimento de CAC. Uma atenção maior deve ser direcionada ao acompanhamento nutricional de pacientes oncológicos, de forma a atuar em uma melhor resposta ao tratamento, 
prevenindo o desenvolvimento de CAC e complicações, e buscando melhoria na qualidade de vida desses indivíduos desde o início do tratamento.

\section{REFERÊNCIAS}

1. INCA. Estimativa - 2018 incidência de câncer no Brasil. Rio de Janeiro: Instituto Nacional de Câncer José Alencar Gomes da Silva (INCA); 2017.

2. Canada. Canadian cancer statistics. Can Cancer Soc. 2017;1-142.

3. Siegel RL, Miller KD, Jemal A. Cancer statistics - 2017. CA Cancer J Clin. 2017;67(1):7-30.

4. Baracos VE, Martin L, Korc M, Guttridge DC, Fearon KCH. Cancer-associated cachexia. Nat Rev Dis Primers. 2018;4:17105.

5. Porporato PE. Understanding cachexia as a cancer metabolism syndrome. Oncogenesis. 2016;5:e200.

6. Fearon K, Strasser F, Anker SD, Bosaeus I, Bruera E, Fainsinger $\mathrm{RL}$, et al. Definition and classification of cancer cachexia: an international consensus. Lancet Oncol. 2011;12(5):489-95.

7. Antoun S, Baracos VE, Birdsell L, Escudier B, Sawyer MB. Low body mass index and sarcopenia associated with dose-limiting toxicity of sorafenib in patients with renal cell carcinoma. Ann Oncol. 2010;21(8):1594-8.

8. Martin L, Senesse P, Gioulbasanis I, Antoun S, Bozzetti F, Deans C, et al. Diagnostic criteria for the classification of cancerassociated weight loss. J Clin Oncol. 2015;33(1):90-9.

9. Kazemi-Bajestani SM, Mazurak VC, Baracos V. Computed tomography-defined muscle and fat wasting are associated with cancer clinical outcomes. Semin Cell Dev Biol. 2016;54:2-10.

10. Muscaritoli M, Lucia S, Farcomeni A, Lorusso V, Saracino $\mathrm{V}$, Barone $\mathrm{C}$, et al. Prevalence of malnutrition in patients at first medical oncology visit: the PreMiO study. Oncotarget. 2017;8(45):79884-96.

11. American Society of Clinical Oncology (ASCO). Managing your weight after a cancer diagnosis: a guide for patients and families. Am Soc Clin Oncol - CancerNet [Internet]. 2014;16. [cited 2019 Sep 3]. Available from: http://www.cancer.net/sites/ cancer.net/files/weight_after_cancer_diagnosis.pdf

12. Arends J, Bachmann P, Baracos V, Barthelemy N, Bertz H, Bozzetti F, et al. ESPEN guidelines on nutrition in cancer patients. Clin Nutr. 2017;36(1):11-48.

13. Omlin A, Blum D, Wierecky J, Haile SR, Ottery FD, Strasser F. Nutrition impact symptoms in advanced cancer patients: frequency and specific interventions, a case-control study. J Cachexia Sarcopenia Muscle. 2013;4(1):55-61.

14. Ezeoke CC, Morley JE. Pathophysiology of anorexia in the cancer cachexia syndrome. J Cachexia Sarcopenia Muscle. 2015;6(4):287-302.

15. Martin L, Watanabe S, Fainsinger R, Lau F, Ghosh S, Quan H, et al. Prognostic factors in patients with advanced cancer: use of the patient-generated subjective global assessment in survival prediction. J Clin Oncol. 2010;28(28):4376-83.

16. Blum D, Omlin A, Baracos VE, Solheim TS, Tan BHL, Stone $P$, et al. Cancer cachexia: a systematic literature review of items and domains associated with involuntary weight loss in cancer. Crit Rev Oncol Hematol. 2011;80(1):114-44.

17. Farhangfar A, Makarewicz M, Ghosh S, Jha N, Scrimger R, Gramlich L, et al. Nutrition impact symptoms in a population cohort of head and neck cancer patients: multivariate regression analysis of symptoms on oral intake, weight loss and survival. Oral Oncol. 2014;50(9):877-83.

18. Ottery FD. Definition of standardized nutritional assessment and interventional pathways in oncology. Nutrition. 1996;12(1 Suppl):S15-9.

19. Kubrak C, Olson K, Baracos VE. The head and neck symptom checklist $(\mathrm{C}$ : an instrument to evaluate nutrition impact symptoms effect on energy intake and weight loss. Support Care Cancer. 2013;21(11):3127-36.

20. Platek ME. The role of dietary counseling and nutrition support in head and neck cancer patients. Curr Opin Support Palliat Care. 2012;6(4):438-45.

21. Nicolini A, Ferrari P, Masoni MC, Fini M, Pagani S, Giampietro $\mathrm{O}$, et al. Malnutrition, anorexia and cachexia in cancer patients : a mini-review on pathogenesis and treatment. Biomed Pharmacother. 2013;67(8):807-17.

22. Kubrak C, Martin L, Gramlich L, Scrimger R, Jha N, Debenham $\mathrm{B}$, et al. Prevalence and prognostic significance of malnutrition in patients with cancers of the head and neck. Clin Nutr. 2020;39(3):901-9.

23. Lotici T, Antunes LBB, Melhem ARF, Bennemann GD, Schiessel DL, Bianca L, et al. Prevalência de perda de peso, caquexia e desnutrição, em pacientes oncológicos. Rev Uniabeu. 2014;17(17):107-24.

24. Rocha LA, Cavagnari MAV, Freitas Melhem AR, Bennemann GD, Antunes LBB, Gavarrete D, et al. Incidência de caquexia, anemia e sintomas de impacto nutricional em pacientes oncológicos. Mundo da Saude. 2016;40(3):353-61.

Local de realização do estudo: Universidade Estadual do Centro Oeste (UNICENTRO), Guarapuava, PR, Brasil.

Conflito de interesse: Os autores declaram não haver.

Trabalho apresentado no XXIII Congresso Brasileiro de Nutrição Parenteral e Enteral, realizado em Foz do Iguaçu, no período de 20 a 23 de outubro de 2019. 